\title{
Glucose Analyzer Based on Self-made Biosensor for High- performance Glucose Detection
}

\author{
Qiang Tan ${ }^{1}$, Cuimei $\mathrm{Bo}^{1, *}$, Jun $\mathrm{Li}^{1, *}$, Yiqing Wang ${ }^{1}$, Xiaorong Wang ${ }^{1}$, Shubo Jiang ${ }^{1}$ \\ ${ }^{1}$ College of Electrical Engineering and Control Science, Nanjing Tech University, Nanjing, China
}

\begin{abstract}
The concentration analyzer with high precision and wide range is the core device for monitoring the fermentation process. In this work, we designed and proposed a lowcost three-electrode glucose analyzer based on a self-made screen-printed enzyme biosensor chip, which has a Prussian blue (PB) nanocubic structure and leads to high sensitivity of $117.31 \mu \mathrm{AmM}^{-1} \mathrm{~cm}^{-2}$. The hardware design of the glucose analyzer can be divided into five critical parts, including digital, signal treatment system, power supply, motor-driven and the host computer. The signal treatment system is used to collect, convert and amplify the weak current signal generated by the biosensor. The digital circuit of the central processing unit of the analyzer is designed using the STM32F407ZET6. Besides, an external analog-to-digital converter is used to achieve high precision $\mathrm{A} / \mathrm{D}$ conversion. The stability of the potentiostat is ensured by designing the precision power supply, hardware filtering, and algorithm filtering. The experimental results show that the glucose analyzer has a wide linear detection range from $1 \mathrm{~g} / \mathrm{L}$ to $120 \mathrm{~g} / \mathrm{L}$ and the coefficient of variation at $1 \mathrm{~g} / \mathrm{L}$ is 0.038 , which exhibits excellent performance in stability and detection accuracy. The analyzer can be applied in the future for in-situ measurement of glucose concentration for its wide-range and high-precision detection capabilities.
\end{abstract}

\section{Introduction}

In order to obtain high efficiency with low cost, the continuous operation dominates the industrial operation in large-scale manufacturing, which strongly relies on the real-time control of the microbial reaction to realize an optimum transfer rate. Therefore, online monitor technology is the key to guide the operation by receiving dynamic fluctuation of the essential parameters[1]. Currently, there have been already some parameters showing the environmental state in the fermentation reactor can be online detected, such as temperature, $\mathrm{pH}$ value, pressure and concentration of dissolved oxygen[2]. However, the real-time detection of constituent concentration, such as glucose, which is directly served as a common substrate to associate with the reaction kinetics in fermentation, has been paid rare attentions to the development of testing instruments.

In recent decades, the application of enzyme biosensors in biosensing has been widely investigated and achieved significant progress[3-5]. Due to the inherent substrate specificity of enzymes and their ability to operate under mild conditions, enzymes have become a biocatalytic pathway, which has caused great research interest [6]. The enzyme biosensor is made by combining a biological enzyme with various electrochemical electrodes or directly fixing the biological enzyme on a substrate electrode[7]. The glucose enzyme biosensor [8-11] have become essential analytical tools in the fields of clinical analysis, environmental monitoring and food safety due to their fast response, high accuracy and low cost [12].

In enzyme biosensors, the properties of electrolytes, membranes, electrodes, and microorganisms change over time[13]. Besides, to achieve high-precision applications and be able to process tiny signals, the noise should be controlled at an extremely low level. In this work, a lowcost three-electrode glucose analyzer based on a selfmade screen-printed enzyme biosensor chip is developed, which includes the design of signal treatment system, digital circuit, precision power supply circuit, motor drive circuit and the host computer. The reliability and stability of the system can be ensured by the precise power supply and stable detection environment. The response current of the enzyme electrode is decreased after long-term use, the system automatically calibrates and establishes the concentration-response equation to eliminate the error. The experimental results show that glucose analyzer has a wide linear detection range.

\section{2 self-made biosensor chip}

In previous work by our team [14-16], PB enzyme biosensor chips with nanocubic crystals were manufactured in batches by screen printing technology for the general detection of various physiological substances. As shown in Fig.1, two solutions were prepared as follows: solution $\mathrm{A}, 0.01 \mathrm{M} \mathrm{K} 4[\mathrm{Fe}(\mathrm{CN}) 6]+$

\footnotetext{
Corresponding author: 1j_bcm@163.com
} 
$0.1 \mathrm{M} \mathrm{KCl}+0.1 \mathrm{M} \mathrm{HCl}$, and solution $\mathrm{B}, 0.01 \mathrm{M} \mathrm{FeCl} 3+$ $0.1 \mathrm{M} \mathrm{KCl}+0.1 \mathrm{M} \mathrm{HCl}$. The syringe was fixed to the automatic syringe pump at a rate of $1000 \mathrm{~L} / \mathrm{min}$ after solution A and solution B were poured into the syringe. At the same time, a beaker at the heating magnetic stirrer was used for reaction vessel, in which the beaker contained distilled water before the start of the reaction. A constant temperature water-bathing was used to obtain the most suitable nanostructure of $\mathrm{PB}$. The PB slurry was obtained by centrifuging PB solution at the beaker, and the mixed ink was formed by mixing the PB slurry with the carbon ink. Then, the PB slurry was screen-printed as a working electrode(WE), a counter electrode(CE) and a connector were generated using carbon ink, a reference electrode (RE) was printed using silver chloride ink. Commercial PVC boards were invoked as the substrate for screen printing. A glutaraldehyde cross-linking method was utilized to fix glucose oxidase (GOD) on the working electrode, and a three-electrode enzyme biosensor chip was finally fabricated.

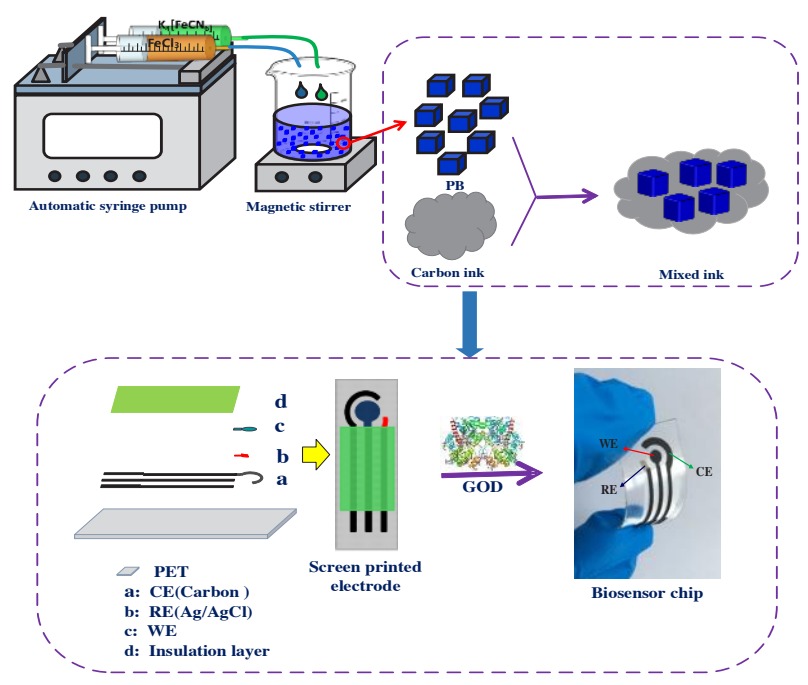

Fig.1.Preparation of biosensor chip

The excellent performance of the self-made biosensor chip has been proved by a series of tests. Fig.2(a) showed the CV diagrams of the biosensor, and the scan rate was $0.05 \mathrm{~V} \mathrm{~s}^{-1}$, hundredth redox peak current is still able to achieve $94.5 \%$ of the first, indicating that the chip has a very excellent repeatability. Fig.2(b) showed the interference of ascorbic acid (AA) and uric acid (UA) on the responses of the glucose biosensor in $0.05 \mathrm{M}$ PBS containing $0.1 \mathrm{M} \mathrm{KCl}$. Fig.2(c) showed the sensitivity of 10 chips randomly selected for glucose. Fig.2(d) showed the sensitivity changes over time, and the chip is tested for performance every 5 days and stored at $4{ }^{\circ} \mathrm{C}$ in air. The self-made enzyme biosensor chip has excellent selectivity, reproducibility and stability at a low operating potential of $-0.05 \mathrm{~V}$.
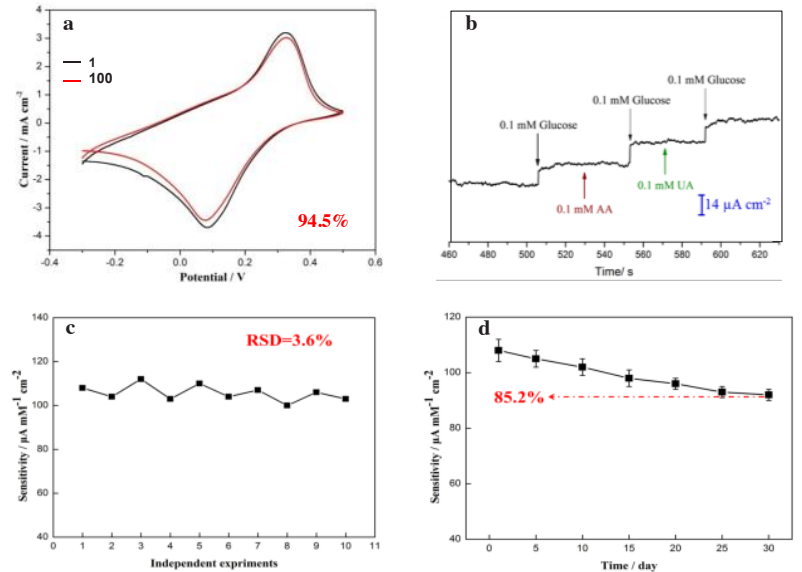

Fig. 2. Performance tests of self-made biosensor chip.

\section{Design of analyzer}

\section{1 hardware design}

Hardware can be divided into five parts including digital design, power supply, motor-driven, the host computer and signal treatment system,. The first is the power supply, the stability of the power supply is under a massive impact on the operation of the entire analyzer. Second, the digital design is mainly based on the STM32F407ZET6 microcontroller unit (MCU), which has the functions of data storage and processing, control of the stirring motor and communication. ADS8332 chip is utilized to achieve 16-bit high-precision analog-todigital conversion. Followed by the design of the motor drive circuit. The glucose measurement needs to be undertaken in a test cell equipped with PBS. A magnetic stirring motor is required to mix PBS and glucose solution; discharge the waste solution after the detection is completed. Fourth is the host computer for humancomputer interaction. RS-485 circuit is designed for communication between the host computer and the MCU. The host computer can display all the information in the detection process and control the glucose analyzer. The most critical signal treatment system will be described in detail in the next section. As shown in Fig.4.All hardware is printed into a circuit board as a more stable and long-lasting solution.

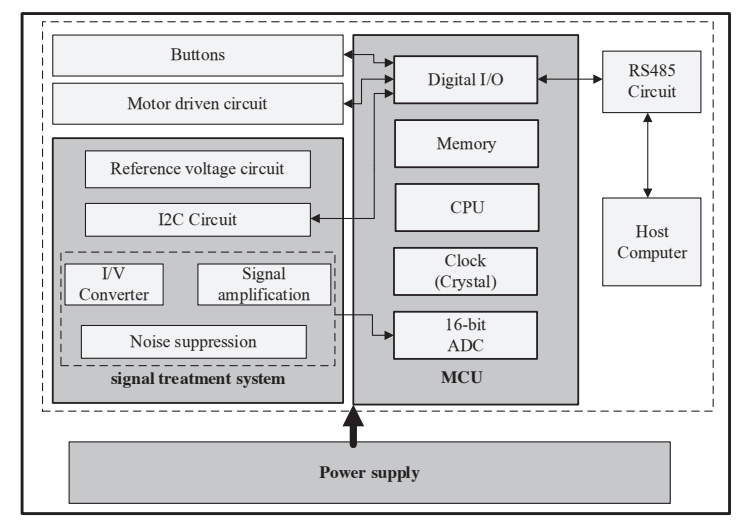

Fig.3. Hardware block diagram. 


\subsection{Signal treatment system}

In a three-electrode system, the RE provides a reference voltage and the working WE promote the electrochemical reaction of the measured solution under the effect of the external excitation signal and then, measures the current formed on the electrode. Meanwhile, the CE forms a circuit with the WE. The measured solution is excited by a voltage signal to produce a redox reaction and generate a weak current. The value of the formed current depends on the concentration of the measured substance. Hence, the concentration of the measured substance can be calculated based on this relation. When performing electrochemical detection, a constant potential should be added between WE and RE to maintain the electrochemical stability of the biosensor.

We used an electrochemical analog front-end chip to build a signal treatment system for this glucose analyzer. The schematic of the simplified circuit is shown in Fig.3. The internal control amplifier A1 of the LMP91000 constitutes a potentiostatic circuit, and the error signal is amplified and fed back to the CE of the biosensor. The impedance change between the $\mathrm{WE}$ and the RE will cause the changes in the voltage of $\mathrm{CE}$, which ensures the stability of the voltage between the RE and the WE. The output voltage of the transimpedance amplifier(TIA) connected to the WE is proportional to the working current of the biosensor. Meanwhile, the WE is connected to the virtual ground (internal ground) through the TIA. The MCU communicates with the LMP91000 through the $\mathrm{I} 2 \mathrm{C}$ interface, which can change the gain of the internal transimpedance RTIA and can also select the reference voltage and bias voltage of the constant potential amplifier. The I2C interface, which can change the gain of the internal transimpedance $\mathrm{R}_{\text {TIA }}$ and can also select the reference voltage and bias voltage of the constant potential amplifier.

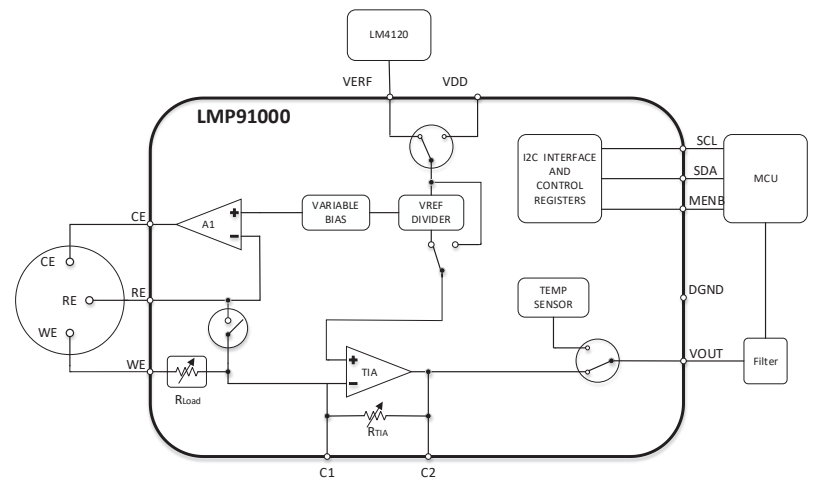

Fig.4.Preparation of biosensor chip

\subsection{Algorithm filtering}

High-precision 16-bit ADC was used for analog-todigital conversion and the ADC sampling period was set to $10 \mathrm{~ms}$. A high-performance first-order inertial filtering was adopted to filter out the interference of random signals. The formula is as follows:

$$
Y(n)=\frac{\alpha X(n)+(K-\alpha) Y(n-1)}{K}
$$

where $\mathrm{K}$ is the magnification of the coefficient $\alpha, \alpha$ is an integer from 0 to $K, X(n)$ is the actual $A D$ value collected this time, $\mathrm{Y}(\mathrm{n}-1)$ is the $\mathrm{AD}$ value obtained after the last filtering, and $\mathrm{Y}(\mathrm{n})$ is the $\mathrm{AD}$ value obtained through this filtering.

In terms of the first-order inertial filtering, we used the $A D$ value collected this time to weight the $A D$ value received last time to obtain a valid filter value, which leads to the previous output signal can effectively be fed back to the next signal. The algorithm filtered out the interference with the tiny signals in the system and obtained accurate $\mathrm{AD}$ values related to the glucose concentration, despite a delay of about $1 \mathrm{~s}$ in the acquisition of concentration information. As presented in Fig.5. Different values of a are used to compare the filtering effect, the sampling period of the ADC was $10 \mathrm{~ms}$. The $\mathrm{AD}$ value collected during a test was converted into a corresponding voltage value $(\mathrm{mV})$. First-order inertial filtering was used for simulation in MATLAB. K was 256.

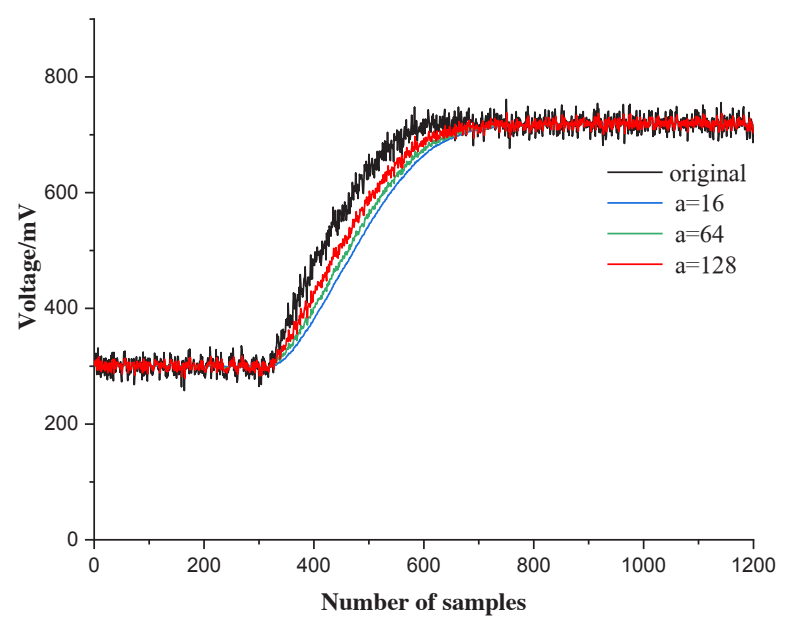

Fig.5. Test of first-order inertial filtering. a is $\alpha$ of formula 1 .

\subsection{Calibration}

The sensitivity of the biosensor chip will decrease with time, and the response current will decrease. This requires the system to calibrate and establish a concentration-response equation to eliminate the error. The glucose concentration is proportional to the response current (or voltage) in the linear detection range of the electrochemical enzyme biosensor. Thus, a known concentration of a standard glucose solution can be used to calibrate this electrochemical enzyme biosensor, which can be explained by the following concentrationresponse equation:

$$
y=\frac{A D V_{0}-A D V_{1}}{C_{0}} x+A D V_{0}
$$


where $\mathrm{ADV}_{0}$ is the $\mathrm{AD}$ value of the solution injected into the PBS which contains no glucose, $\mathrm{ADV}_{1}$ is the $\mathrm{AD}$ value of the standard glucose solution of known concentration injected into $\mathrm{PBS}, \mathrm{C}_{0}$ represents the concentration value of the standard glucose solution, $y$ is the $\mathrm{AD}$ value of the solution injected into the PSB of which the glucose concentration is unknown, and $\mathrm{x}$ is the glucose solution concentration to be measured.

\section{Experiments and results}

At room temperature, the biosensor chip was placed in a test cell with $3 \mathrm{~mL}$ of PBS solution in it. As presented in Fig.6. $5 \mathrm{uL}$ of PBS buffer was injected into the test cell to obtain ADV0 after turning o $\mathrm{n}$ the machine and activating the biosensor chip. Secondly, drained the waste solution from the test cell, cleaned the test cell, inject $3 \mathrm{~mL}$ of PBS solution again, and finally injected $5 \mathrm{uL}$ of glucose solution $(60 \mathrm{~g} / \mathrm{L})$ to obtain the value of ADV1. The concentration characteristic equation was established according to the calibration formula, and thus the calibration of the biosensor chip was completed. To ensure the calibration was correct, we repeat the second step of injecting $5 \mathrm{uL}$ of glucose solution $(60 \mathrm{~g} / \mathrm{L})$. If the testing result is $2 \%$ below or above the $60 \mathrm{~g} / \mathrm{L}$ concentration, the calibration is successful. Otherwise, recalibrate.

When the calibration finished, a linearity test was conducted by using a glucose solution of known concentration. The measured increment was: 1) $0.2 \mathrm{~g} / \mathrm{L}$ under the concentration from 1 to $2 \mathrm{~g} / ; 2$ ) $2 \mathrm{~g} / \mathrm{L}$ under the concentration from 2 to $10 \mathrm{~g} / \mathrm{L} ; 3) 10 \mathrm{~g} / \mathrm{L}$ from $10 \mathrm{~g} / \mathrm{L}$ to $110 \mathrm{~g} / \mathrm{L}$; and $20 \mathrm{~g} / \mathrm{L}$ form 110 to $120 \mathrm{~g} / \mathrm{L}$. Each increment was tested five times and averaged. Electrolytes, membranes, electrodes and microorganisms change their properties overtime during the test. In this work, we recalibrated every hour to eliminate this error. The final result is shown in Fig. 7.

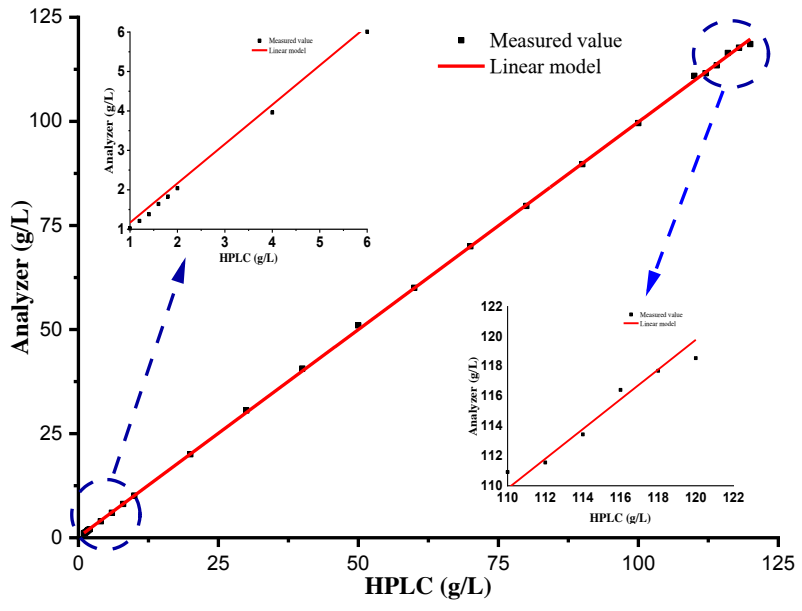

Fig.7. Linearity test of glucose analyzer

The linear regression equation for calculating the concentration (x) measured by HPLC and the concentration (y) measured by the instrument, $\mathrm{y}=$ $0.9967 x+0.1708, R 2=0.999$. The experimental results showed that the analyzer has good linearity between the glucose concentration of $1-120 \mathrm{~g} / \mathrm{L}$. The analyzer fully utilizes the linear measurement capability of the selfmade enzyme biosensor. The motor had been rotating at an appropriate speed in the process of measurement to prevent the start and stop of the stirring motor from affecting the detection environment.

$1 \mathrm{~g} / \mathrm{L}$ and $60 \mathrm{~g} / \mathrm{L}$ were selected for stability testing which was shown in Fig.8. Each concentration was tested three times a day for 21 consecutive days with other conditions unchanged. In the continuous stability test, the analyzer and the self-made biosensor showed outstanding stability. 63 tests were carried out on $1 \mathrm{~g} / \mathrm{L}$ glucose and the average measured concentration was $0.988 \mathrm{~g} / \mathrm{L}$, the coefficient of variation was 0.038 . The analyzer has a high measurement accuracy even at low levels. Meanwhile, $60 \mathrm{~g} / \mathrm{L}$ was used for testing and the mean measured concentration was $59.57 \mathrm{~g} / \mathrm{L}$, the coefficient of variation was 0.01 .

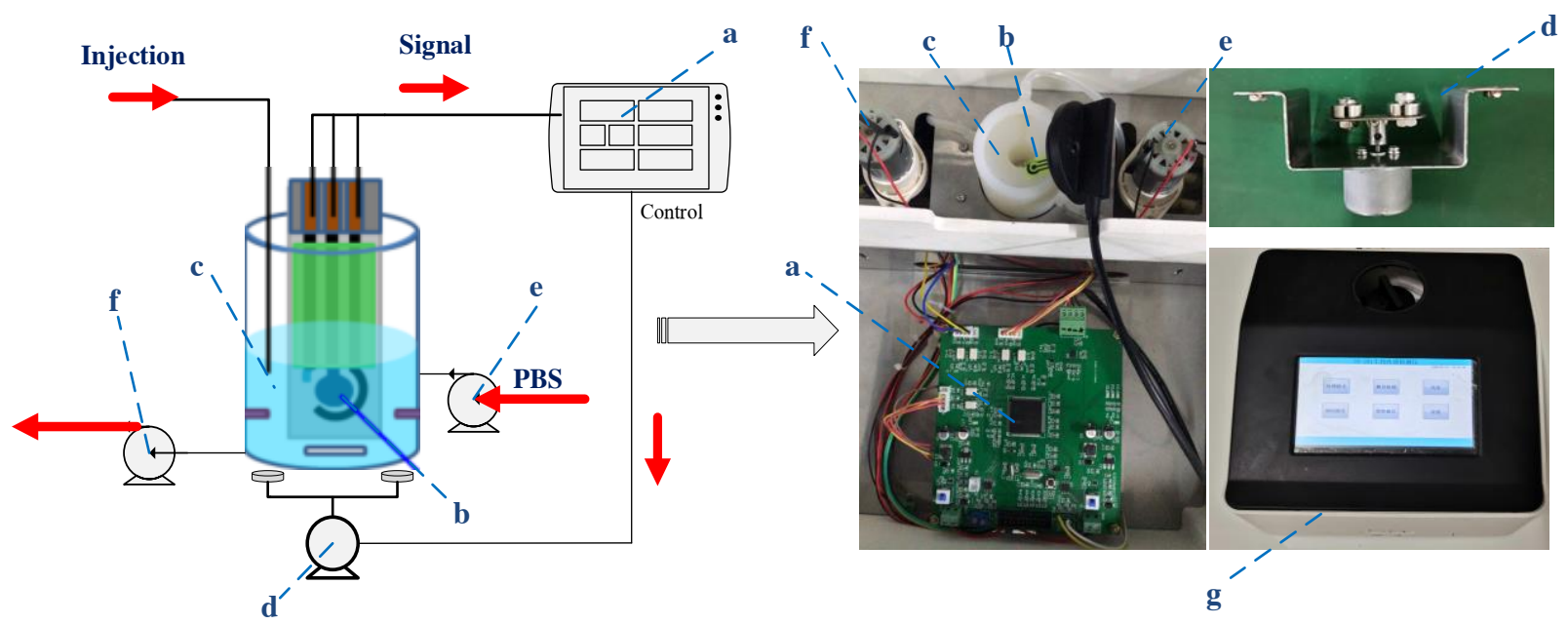

Fig.6. Analyzer structure. (a) Hardware, (b) self-made glucose biosensor chip, (c) test cell, (d) Magnetic stirring motor, (e) Inlet pump, (f) Outlet pump, (g) Overall image of the instrument. Each time Before testing, wash the test cell with PBS, pump 3mL PBS through the peristaltic pump, and then inject glucose solution for concentration detection. After the test, the Waste solution is discharged through the pump. 

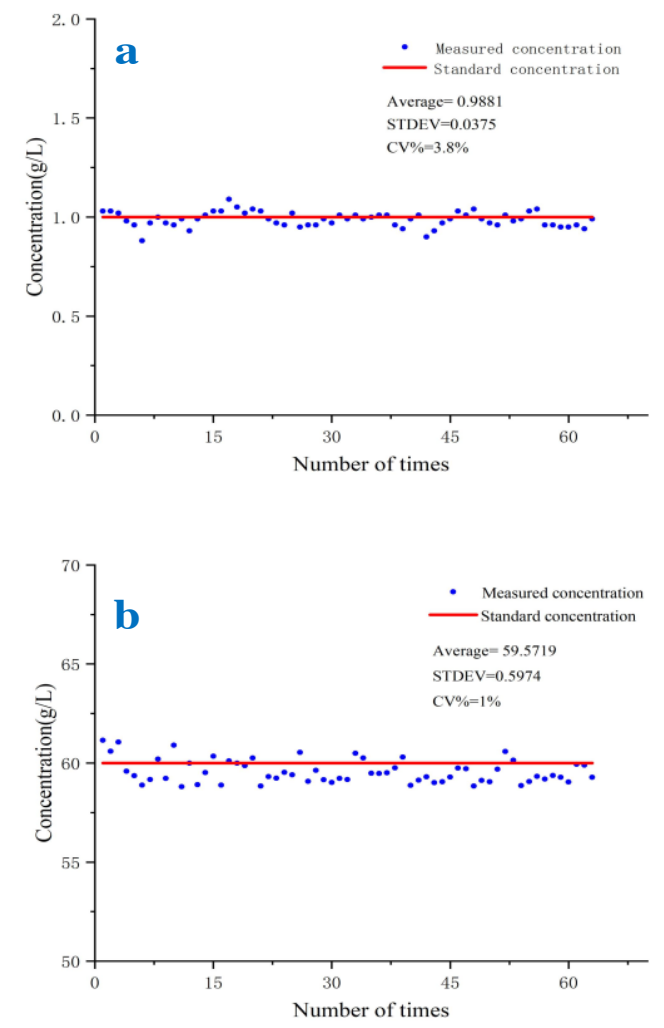

Fig.8. Stability test of glucose analyzer. (a)test with $1 \mathrm{~g} / \mathrm{L}$ of glucose (b) test with $60 \mathrm{~g} / \mathrm{L}$ of glucose

\section{Conclusions}

In this work, we designed a glucose analyzer based on self-made biosensor chips. The analyzer has a wide linear detection capability of $1-120 \mathrm{~g} / \mathrm{L}$ in the detection environment of PBS; the $\mathrm{CV}$ at $1 \mathrm{~g} / \mathrm{L}$ is 0.038 . The glucose analyzer has excellent stability and measurement accuracy. This glucose analyzer is quite promising for in-situ on-line detection of glucose concentration under various conditions, especially in the fermentation process.

\section{Acknowledgements}

This work was financially supported by National Natural Science Foundation of China (No. 2172780038 ), open subject of State Key Laboratory of Materials Chemistry Engineering ().

\section{References}

1. Lisa Mears, Stuart M. Stocks, Gürkan Sin, Krist V. Gernaey, A review of control strategies for manipulating the feed rate in fed-batch fermentation processes, J. Biotechnol, 245, 34 (2017)

2. Xuzhi Zhang, Xiaoyu Jiang, Zhihui Hao, Keming $\mathrm{Qu}$, Advances in online methods for monitoring microbial growth, Biosens. Bioelectron, 126, 433(2019)

3. Elif Burcu Bahadır, Mustafa Kemal Sezgintürk, Applications of commercial biosensors in clinical, food, environmental, and biothreat/biowarfare analyses, Anal. Biochem, 478, 107 (2015)

4. L.C. Clark, C. Lyons, Electrode systems for continuous monitoring in cardiovascular surgery, Ann. N. Y. Acad. Sci, 102, 29 (1962)

5. Conan Mercer, Richard Bennett, Peter Ó. Conghaile, James F. Rusling, Dónal Leech, Glucose biosensor based on open-source wireless microfluidic potentiostat, Sens. Actuators B, 290, 616 (2019)

6. J. Wang, Electrochemical detection for microscale analytical systems: a review, Talanta, 56, 223 (2002)

7. F. Arduini, C. Zanardi, S. Cinti, F. Terzi, D. Moscone, G. Palleschi, R. Seebe, Effective electrochemical sensor based on screen-printed electrodes modified with a carbon black-Au nanoparticles composite, Sens. Actuators B, 212 , 536 (2015)

8. S. Calabrese Barton, J. Gallaway, P. Atanassov, Enzymatic biofuel cells for implantable and microscale devices, Chem. Rev 104, 4867 (2004)

9. D. Leech, P. Kavanagh, W. Schuhmann, Enzymatic fuel cells: recent progress, Electrochim. Acta, 84, 223 (2012)

10. J. Wang, Carbon-nanotube based electrochemical biosensors: a review, Electroanalysis, 17, 7(2005)

11. Ievgen Mazurenko, Vivek Pratap Hitaishi, Elisabeth Lojou, Recent advances in surface chemistry of electrodes to promote direct enzymatic bioelectrocatalysis, Current Opinion in Electrochemistry 19, 113 (2020)

12. Xiuling MAO, Jian WU, Yibin YING, Application of Electrochemical Biosensors in Fermentation, Chin. J. Anal. Chem. 36, 1749(2008)

13. Turner, Robin FB, D. J. Harrison, and HENRY P. Baltes, A CMOS potentiostat for amperometric chemical sensors, IEEE J. Solid-State Circuits, 22.3, 473 (1987).

14. Lifang Liu, Lei Shi, Zhenyu Chu, Jingmeng Peng, Wanqin Jin, Prussian blue nanocubes modified graphite electrodes for the electrochemical detection of various analytes with high performance, Sens. Actuators B, 202, 820 (2014)

15. Danfeng Jiang, Zhenyu Chu, Jingmeng Peng, Wanqin Jin, Screen-printed biosensor chips with Prussian blue nanocubes for the detection of physiological analytes, Sens. Actuators B, 228, 679 (2016)

16. Linlin Li, Jingmeng Peng, Zhenyu Chu, Danfeng Jiang, Wanqin Jin, Single layer of graphene/Prussian blue nano-grid as the lowpotential biosensors with high electrocatalysis, Electrochimica Acta, 217, 210 (2016) 